\title{
Figural goodness and internal structure in perceptual discrimination
}

\author{
FRED L. ROYER ${ }^{1}$ \\ BRECKSVILLE VA HOSPITAL, BRECKKSVILLE, OHIO
}

The time required for 24 Ss to sort four decks of cards composed of 5-dot figures (Garner \& Clement, 1963) was measured. A deck consisted of 32 cards: 4 each of 8 patterns. There were 4 sets of figures: Sets $A 1$ and $A 2$ had identical amount and form of redundancy; so also did B1 and B2. Sets $\mathrm{Bl}$ and $\mathrm{B} 2$, having more uncertainty in simple contingencies and having negative interaction terms, required significantly more time to sort than Sets A1 and A2. All sets differed in mean ratings of figural gondness, (Garner \& Clement, 1963). Even when amount and form of redundancy are held constant, the figural goodness of the individual figures constituting the set influence the discriminability. Sets consisting of good or simpler figures are easier to sort.

Garner (1962), in his review of pattern perception, distinguished between the effects of the amount and form of redundancy in pattern perception. He interpreted the reported improved performance in the discrimination of symmetrical patterns as resulting not from redundancy but form of redundancy (p. 192). The amount of redundancy is determined by the number of stimuli selected from the total set. Thus, if two subsets of equal size are drawn from a total set of all figures which could be generated, given certain variables, the amount of redundancy would be the same. The different rules by which the two sets are drawn to produce symmetry or asymmetry will affect the form of redundancy.

It is possible, however, to generate a set of figures which will have the same amount of redundancy and the same form of redundancy but which will vary in Gestalt characteristics. Such figures can be generated from the use of dots placed in a 3 by 3 matrix. Garner and Clement (1963) used such figures in their study of the relationship between size of inferred subset and figural goodness.

Each cell of the matrix may be considered as a binary variable (having a dot or no dot). Given any subset of the total $\mathbf{5 1 2}$ figures constituting the total set, the particular distribution of the redundancy can be described. That subset will be one of a large number of other subsets of equal size having identical distributions of redundancy. In other words, the relative positions of the variables across all figures may be changed but the information measure will be unaffected. In the case of dot figures, however, such rearrangement of variables will result in quite different looking figures. If the matrices were large enough one might end up with figures which resembled cows, houses or circles in one subset but which resembled people, trees and automobiles in another, yet the sets would have identical information measures. In other words, in a discrimination task the available amount and form of redundancy might be ignored in favor of some other characteristic of the figures.

The purpose of this experiment was to evaluate the contribution of differences in Gestalt to discriminability of visual figures when the amount and form of redundancy is controlled. For this purpose the figures used by Garner and Clement (1963) are ideal since ratings of figural goodness (on a scale of 1 to 7 ) are known for each of 90 5-dot figures satisfying the criterion of at least one dot in each row and column of an imaginary 3 by 3 matrix (personal communication). Figural goodness is a Gestalt characteristic which therefore can be manipulated independently of the amount and form of redundancy of the subset used.

\section{Method}

Subjects. Twenty-four students of a course in general psychology at the Kent State University ${ }^{1}$ served as Ss; nine were men, 15 were women.

Selection of Figures. Two subsets of eight figures each were selected from those of Garner and Clement (1963, personal communication). In drawing the first subset, the eight different patterns having the best goodness-of-form ratings were chosen after excluding two having extremely limited possibilities for transposition into other Garner-Clement patterns. The second subset was composed of the figure having the poorest rating and the seven other figures which were equivalent by operations of rotation and reversal. These figures were then subjected to a variety of transpositions of variables; e.g., columns and rows were transposed in order. Mean goodness ratings of the derived subsets were calculated; those two derived subsets having the most similar mean ratings were chosen. All these patterns are shown in Fig. 1.

As finally constituted, there were four subsets of eight figures each. For purposes of clarity these will hereafter be referred to as sets; it will be understood that they are subsets of the total set of 512 figures. Two of these sets, Sets A1 and A2, represented the extremes with respect to mean ratings of goodnessof-form, which were 1.96 and 5.49 , respectively. The mean ratings of goodness-of-form of the remaining two sets, Sets $\mathrm{A} 2$ and $\mathrm{B} 1$, are closer to one another than they are to the mean ratings of the related figures from which they were generated by transposition of the 


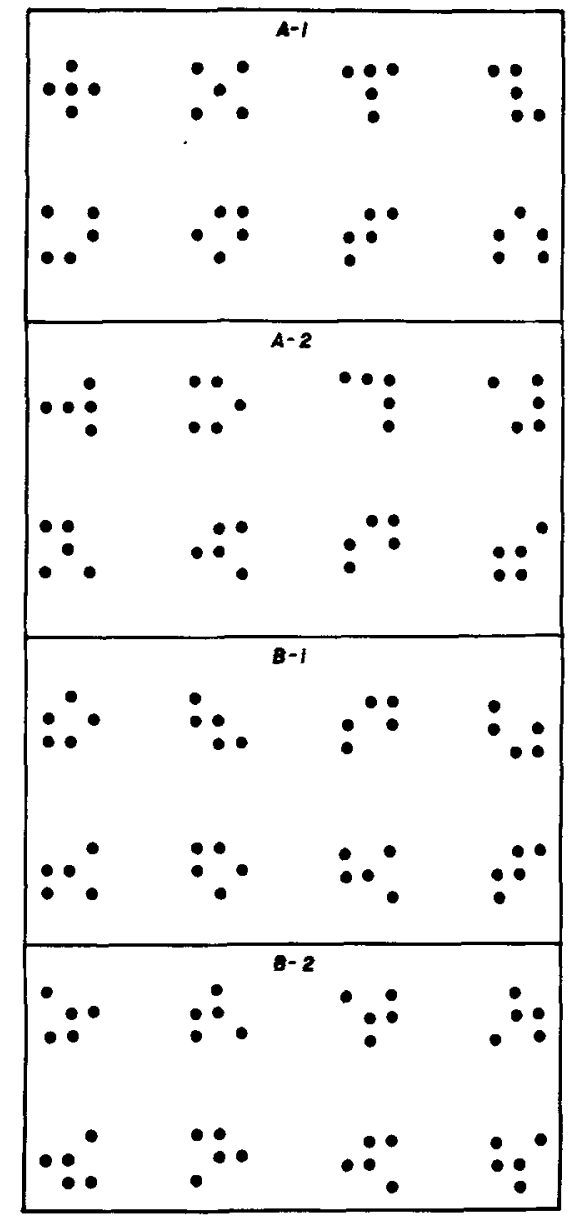

Fig. 1. Sets of 5-dot figures used in the sorting task.

columns to orders $1-3-2$ and 2-1-3 respectively. Set A2 had a mean rating of 3.48 and set $B 1$ had a mean rating of 3.84 .

The sets also differed in the form of redundancy. Ideally, both A- and B-Sets should have had identical amounts of internal structure. Unfortunately, peculiarities of the 5-dot patterns prevented either the use of all variables or equivalent uncertainty per variable; therefore, there is a small difference in amount of internal structure. As a result, amount and form of constraint are confounded between the A- and B-Sets, although not within sets, of course. The relative distribution of uncertainty in the simple contingencies and interaction components are quite different. In Sets $\mathrm{A} 1$ and $\mathrm{A2}$, the total constraint is $5.6160^{\circ}$ bits with 4.1520 in simple contingent uncertainty and 1.4649 bits in interaction uncertainty. Sets B1 and B2 have 5.0000 bits of total constraint, with 7.2079 bits in simple contingent uncertainty and $\mathbf{- 2 . 2 0 7 9}$ bits in interaction uncertainty.

Stimuli. Each of the sets of eight figures were reproduced photographically to fit in a 3 in. $x 3$ in. square. The figures were mounted in two rows on a piece of white poster-board, 22 in. $\times 32$ in. The patterns are reproduced in Fig. 1 in the order in which they occurred. Each transformed figure had the same relative position in the derived setas its parent figure. Thus, position within sets having identical form of redundancy was controlled.

A deck, containing four reproductions of each of the eight figures was constructed by pasting the reproduction on a larger 5 in. $x 5$ in. card. The figure was mounted flush with the left side of the card, providing a space for handling the card and providing unequivocal orientation of the figure.

Procedure. Each $\mathrm{S}$ was brought into the room where it was explained that the task he was about to perform was a card sorting task. A practice set of figures, geometric designs taken from Whitman and Garner (1962), were shown to S. He was handed a deck of cards face down and told to sort themas rapidly and accurately as possible into piles under the figure which the card matched. He was told that when E said "Go!" he was to flip the deck toward him and sort. Any errors in following instructions were pointed out, as were any inaccuracies in sorting. The $S$ was allowed to ask any questions about procedure and then was told that a different type of figure would be used henceforth. He was told that the figures were formed of five dots.

The first set was exposed; 5 sec. later $\mathbf{E}$ handed $\mathbf{S}$ the deck of cards and said "Go!"' as soon as S held the deck firmly in the correct position. E operated a Standard Electric Interval Timer which he started manually and synchronously with this starting signal; he stopped the timer synchronous with the placement of the last card on the table. With each succeeding set, $\mathrm{E}$ instructed $\mathrm{S}$ that the procedure would be identical with the last one and repeated that he was to sort the cards as rapidly and accurately as possible.

Experimental Design. A repeated-measures design was used (McNemar, 1962). All 24 possible orders of presentation of the four different sets of stimuli were used in order to provide counterbalancing for ordereffects. The orders were randomized from a table of random numbers. As Ss arrived, they were assigned consecutively to the orders on the already prepared randomized list. The order of the cards in each deck were randomized by shuffling before S's arrival.

\section{Results}

The mean sorting time for the four sets of eight 5-dot patterns are shown in Fig. 2 as a function of their mean rated goodness-of-form. The ordering of the means is clearly related to the mean goodnessof-form for the set. Set $A 1$, with a mean rating of 1.96 has a mean sorting time of $68.43 \mathrm{sec}$.; Set A2, whose figures have a mean rating 3.48 for goodness-of-form, has a mean sorting time of 89.55 sec. Sets B1 and B2 have mean ratings of goodness-of-form of 3.84 and 5.49 and mean sorting times of 152.77 and 169.04 respectively. An analysis of variance of the sorting 


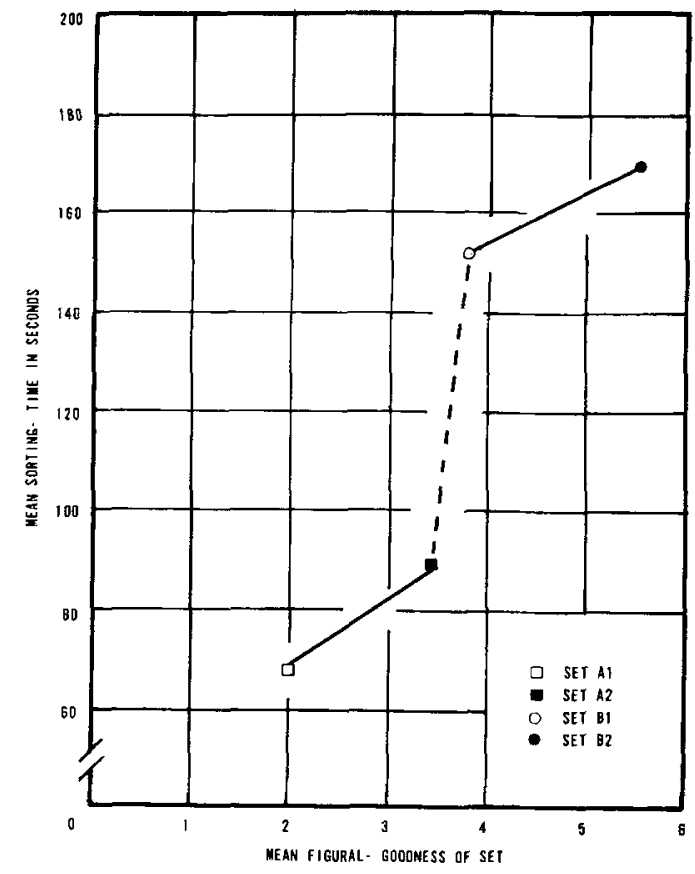

Fig. 2. Mean sorting time of sets as a function of mean judged goodness-of-form.

time, using the repeated measures model, gave an $F=95.85(\mathrm{df}=3 / 69)$ for which $\mathrm{p}<.001$. All differences between pairs of adjacent ordered means were significant at the .05 level.

It may be seen in Fig. 2 that the sorting times of Sets $\mathrm{A} 1$ and $\mathrm{A} 2$, having identical form of redundancy, are quite different from those of Sets $B 1$ and $B 2$, which also have identical form of redundancy. An analysis of variance of the sorting times for sets differing in form of redundancy (comparing Sets A1 and A2 combined, with Sets $\mathrm{B} 1$ and $\mathrm{B2}$ combined) produced an $\mathrm{F}=110.76(\mathrm{df}=1 / 71)$ which has a $\mathrm{p}<.001$.

These results demonstrate that, even where the distribution of the uncertainty is identical as within Sets A1 and A2 or within sets B1 and B2, the goodnessof-form of the figures constituting the set has an effect on the discriminability. In other words, the perceptual simplicity of the single patterns forming the set influences performance. Although the differences in amount and form of internal constraint are confounded, it is clear that the confounded effects on discriminability are quite marked.

\section{Discussion}

Garner (1962) has predicted that increases in redundancy improve discriminability and that a form of redundancy which minimizes the contingent uncertainties between pairs of stimulus variables would be optimal for discrimination. The confounding of amount and form between the A- and B-Sets does not allow one to delineate clearly the source of the sizable dif- ferences observed between the A- and B-Sets, but they are clearly in the direction predicted by Garner. The differences in amount of redundancy are small by comparison to the differences in form of redundancy. It seems reasonable to suppose that most, even if not all, of the differences are attributable to differences in form.

Where the form of the constraint is constant, as between Sets AI and A2 and between Sets B1 and B2, the mean rated goodness-of-form has a definite effect. The significant role which figural goodness plays in the discrimination time poses a problem of interpretation of its effect.

In a sorting task such as the one used here, $\mathrm{S}$ is at liberty to respond either to the properties of the specific subset presented or to the properties of each individual stimulus, and perhaps to both at some time or another. Since the mean rating of figural goodness reflects properties of individual stimuli, it must be presumed that Ss used this property in sorting.

When Garner and Clement (1963) collected their ratings of figural goodness they were testing a hypothesis that where $\mathrm{S}$ views a single stimulus he makes an inference about the size of the subset from which the figure is drawn out of the total set of figures which could be generalized from the variable suggested by the particular stimulus. The ratings were highly correlated with the size of subsets found by grouping together patterns which were identical when rotated or reflected. Ratings were also correlated with the size of groups into which Ss sorted stimuli as similar. Thus, a single stimulus has a subjective or psychological redundancy. How might this psychological redundancy be used?

The total time score obtained when $S$ sorts a deck of cards may be thought of as a cumulation of a series of reaction times. With each stimulus card it is necessary to recognize the figure, remember the location of its matching figure on the board, or proceed directly through a matching procedure successively until the card is identified as being identical to a particular one on the board.

We could expect that any characteristic of the individual stimuli which would enhance its discriminability with respect to others in the set (i.e., decrease confusion) or decrease the number of successive decisions, or facilitate the learning of location on the board, should lead to decreased sorting time.

Handle and Garner (1966), using the same total set of figures from which these were drawn, showed that when Ss produce associates to a given pattern, patterns from larger sized inferred subsets (poorer patterns) have many other patterns as associates while those from smaller sized subsets (good patterns) have very few different associates. The associates were almost completely unidirectional, so that the associates of good figures tend to be those having better form. Those figures having good form in this experiment, then, should be recognized more quickly and with less 
possibility for confusion. Their associates should be more restricted.

The number of alternate figures based upon rotation and reflection may itself play a very large role when $S$ uses figural properties as the basis of sorting. Children frequently demonstrate rotation and reflection errors in learning to write. Bower (1966) has recently demonstrated that two month old babies possess the capacity for shape discrimination under rotational transformation and this "response to a shape invariant is more primary than response to a simple variable such as orientation."

In situations such as that provided by the present experiment, $S$ must discriminate among figures on the basis of orientation, particularly in Set B2. Bower's work suggests that this discrimination must be made on the basis of other cues, perhaps response variables from other systems. Sensory-tonic theorists of perception hold that visual space constancy is achieved by the participation of other modalities in the perceptual process (Bruell, 1966). In other words, the identity of a particular figure in this experiment is not given by the visual system alone, but by the additional correlation of visual information with response variables or other non-visual information such as would define vertical and horizontal axes. Increased sorting times would reflect the "response uncertainty" or number of perceptual alternatives which must be dealt with in order to identify the particulate. Royer and Garner (1966) have shown with auditory temporal patterns that the time required to organize sequences of sounds into a pattern increases with the number of alternative organizations which Ss may make of the sequence.

In summary, the explanation of the results follows this line of reasoning: when $\mathrm{S}$ uses figural properties as the basis of sorting, rather than information in the set of figures presented, he actively perceives stimuli existing in sets and subsets (Garner, 1966); he reacts to the particulate on the basis of his inference about the total set of stimuli. His discrimination processes will be affected, in terms of time, by the associational relation of the particulate to other nonequivalent figures (Handel \& Garner, 1966) and by the size of the subset of equivalent figures from which the particulate must be distinguished according to response variables related to rotation and reversal. Redefinition of the stimulus structure to include information from other modalities may yet show discrimination of these types of stimuli to be consistent with Garner's prediction.

\section{References}

Bower, T. G. R. Slant perception and shape constancy.in infants. Science, 1966, 151, 832-834.

Bruell, J. H. The sensori-motor nature of visual space perception. In S. Wapner \& B. Kaplan (Eds.), Heinz Werner, 1890-1964, Papers in memorium, Clark University Press: Worcester, Mass, 1966. Pp. 11-15.

Gamer, W. R. Uncertainty and structure as psychological concepts. New York: Wiley, 1962.

Garner, W. R. To perceive is to know. Amer. Psychologist, 1966, 21, 11-19.

Gamer, W. R., \& Clement, D. E. Goodness of pattern and pattern uncertainty. J. verbal Learn. verbal Behav., 1963, 2, 446-452.

Handel, S., \& Garner, W. R. The structure of visual pattern associates and pattern goodness. Percept. \& Psychophys., 1966, 11. 33-38.

McNemar, W. Psychological statistics. New York: Wiley, 1962.

Royer, F. L., \& Gamer, W. R. Response uncertainty and perceptual difficulty of auditory temporal patterns. Percept. \& Psychophys., $1966,1,41-47$.

Whitman, J. R., \& Gamer, W. R. Free recall learning of visual figures as a function of form of internal structure, $J$. $e x p$. Psychol., 1962, 64, 558-564.

\section{Note}

1. The author wishes to express his gratitude to Dr. Joseph Grosslight, Chairman, Department of Psychology, Kent State University, for his assistance in obtaining subjects.

(Received in the Editorial Office, July 14,1966.) 